\title{
ENDOSCOPIC EVALUATION OF SINUS MEMBRANE ELEVATION USING MAGIC SINUS LIFTER OSTEOTOME
}

\author{
Ashraf Ghanem* and Hamed Gad**
}

\begin{abstract}
Objectives: The pupose of the study was to visualize the integrity of the sinus membrane by endoscope during sinus lifting via magic sinus lifter.

Methods: Twelve patients with atrophic distal edentulous maxillary region with bone height ranged from 3-5 mm. These patients were selected to perform crestal sinus lifting under endoscopic monitoring with a newly designed sinus lifter (magic sinus lifter). The endoscope was used to visualize the membrane elevation during lifting procedures and implant insertion.
\end{abstract}

Results: Ten cases showed succefull membrane elevation without any tear. Two cases monitored with membrane perforation. One was visualized when the endoscope entered through the crestal osteotomy site, while the other membrane perforation visualized from lateral sinus wall. There was a correlation of the perforated membrane with its morphology as it was irregular shape and thin in thickness. The magic sinus lifter showed marvelous bone cutting and elevation without any heat generation.

Conclusion: Endoscopic visualization of sinus membrane during elevation is an accurate method for membrane assessment. The magic sinus lifter can penetrate the posterior crestal bone to elevate the sinus membrane with gentle malleting. The use of endoscope should be recommended in thin membranes when endoscopy equipment\& training is available.

KEY WORDS: Endoscopy, magic sinus lifter, perforation, crestal technique.

\section{INTRODUCTION}

Implant placement in the posterior maxilla often creates a challenge because of the proximity of the inferior wall of the maxillary sinus and inadequate bone height following the tooth loss. The maxillary sinus augmentation became a prerequisite to provide adequate length of bone to place implants fixture. Augmentation was first reported by Boyne and James ${ }^{1}$ who recommended the lateral window approach which was invasive and carries the risk of Schneiderian membrane perforation. Summers ${ }^{2}$ introduced a less invasive procedure which he

* Associate Professor; Department of Oral \& Maxillofacial Surgery, Faculty of Dentistry, Minia University, Egypt.

** Lecturer; Department of Oral \& Maxillofacial Surgery, Faculty of Dentistry, Minia University, Egypt. 
named the osteotome sinus lift technique (crestal approach). It consists of four calibrated osteotomes used to widen the osteotomy site and elevate membrane at same time. Some cases showed early failure, infection or sinusitis due to maxillary sinus membrane perforation. ${ }^{3}$ Thus there were shift to use short implants ${ }^{4}$ that can be success fully loaded in maxillary bone with a residual bone height of 4-6 $\mathrm{mm}$. Unfortunately their long-term prognosis is unknown, which in turn led researchers to look for a new technique that can bypass these shortcomings. The "Innobiosurg" Company (Korea), offered a specially designed osteotome used for the Schneiderian membrane elevation which is the magic sinus lifter osteotome.

They claimed the ability of the magic lifter osteotome to elevate the sinus membrane from the crestal approach with membrane elevation control without liability of complications as membrane perforation. However, the osteotomy technique is blind and there is no direct vision to the sinus membrane elevation, consequently; the purpose of the study was to use the endoscope with a real magnification from inside the sinus to precisely and accurately evaluate the integrity and safety of the Schneiderian membrane during crestal lifting procedures and implant placement.

\section{PATIENTS AND METHODS}

Twelve patients ( 4 males and 8 females) ranging from 25- 45 years were selected from the Out Patient Clinic of Oral and Maxillofacial Department, Faculty of Dentistry, Minia University Dental Hospital to be included in the study. All patients fufilled inclusion criteria:

Patients free from any systemic or local disease that might negatively affect the implant surgery or complicate the healing process, Free from abnormal habits as bruxim, the edentulous ridge covered with optimal thickness of mucoperiosteum with no signs of inflammation, ulceration or scar tissue,the occlusion showed sufficient inter-arch space for future prosthesis,bone height was ranging 3-5 $\mathrm{mm}$ and crestal bone width at least $4 \mathrm{~mm}$. The Cone beam computerized tomography scan ( CBCT) was performed to evaluate the morphology, thickness of the sinus membrane and the accurate bone dimensions (fig 1).

Mucosal thickening of $>2 \mathrm{~mm}$ was classified according to the criteria adopted from Soikkonen \& Ainamo ${ }^{18}$ :

1. Flat: Shallow thickening without well defined outlines.

2. Semi-spherical: Thickening with well-defined outlines rising in angle of $>30^{\circ}$ from the floor or the walls of the sinus.

3. Mucocele-like: Complete opacification of the sinus.

4. Other mucosal thickening types or pathological findings.

The procedure of closed sinus lifting using the magic sinus lifter was monitored with the endoscope from a lateral small trephined window. Two surgeons were included in the procedure. One surgeons performs the sinus membrane elevation and implant insertion, while the second endoscopically monitors from inside the sinus the first surgeon work.of elevation.

\section{Surgical procedures}

Through the use of CBCT at the proposed implant site. Measurments were taken at the crossed lines to determine the implant site of as well the bone thickness and the morphology of the Schneiderian membrane (Fig. 1). Profound local anesthesia was achieved for all patients using posterior superior alveolar nerve block (PSA) for molar area or infraorbital nerve block for the premolar area and palatal infiltration. The local anesthetic solution $4 \%$ Ubistesin with 1:100000 epinephrine* was used for both buccal and palatal injections.

\footnotetext{
* Ubeistesin Forte 4\% : Articaine 4\% and Adrinaline 1: 100,000, 3M ESPE Co. Germany.
} 
A sharp crestal incision was first performed in the edentulous area. The flap was retracted to expose the crestal and buccal bone. The initial pilot drill was used to locate implant position by penetrating $1 \mathrm{~mm}$ of the crestal cortical bone. A trephine bur on hand rotary drill was used to make a small round window on the buccal wall of the sinus apical to the proposed implant length. The bony window was easily detached from the sinus membrane on the lateral wall by tip of mucoperiosteal elevator (Fig. 2).

The small detached round trephined bone was placed in a bone well and covered by saline solution $0.9 \mathrm{ml}$ to prevent its dryness. The endoscope was introduced inside the sinus from the trephined lateral window performed. The other surgeon performed the closed sinus lifing technique with

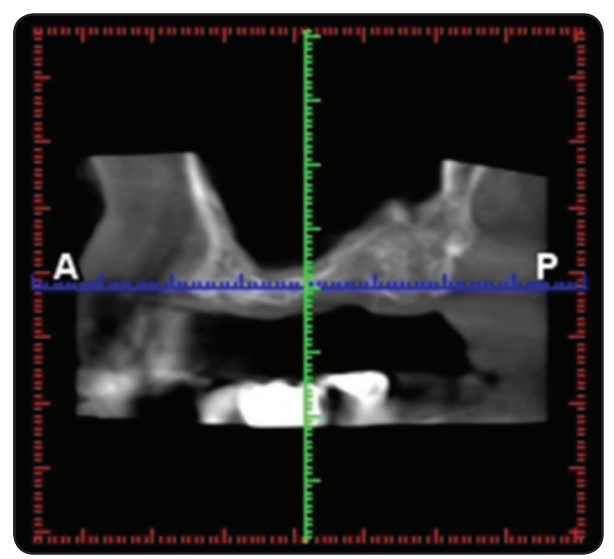

Fig. (1) CBCT showing at the proposed implant site and measurements were taken at the crossed lines .

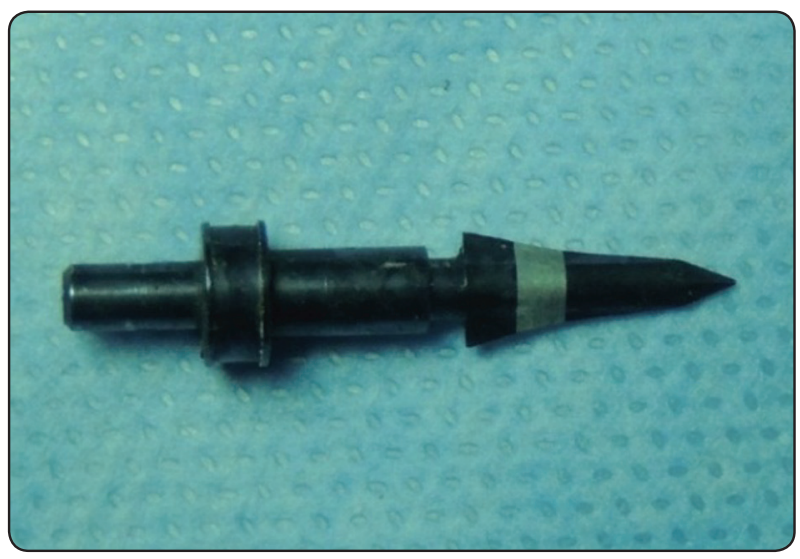

Fig. (3) The Magic Splitter two malleting instruments. First instrument was the magic splitter which is sharp, graduated and can penetrate maxillary posterior bone easily with very gentle malleting. The blade of the magic Splitter was placed on mesiodistal direction, and then it was inserted along with the ridge axis direction.

This blade left about $1 \mathrm{~mm}$ of bone apically to preserve and maintain integrity of the Schneiderian memebrane. Second instrument was the magic sinus lifter has sharp blades with rounded hollow apex to fix the bone with the membrane over it. The magic sinus lifter was placed in the mesiodistal direction (as the previous Magic Splitter) then malleted to lift the remaining part of apical bone fracturing it toward the sinus. (Fig.3-4)

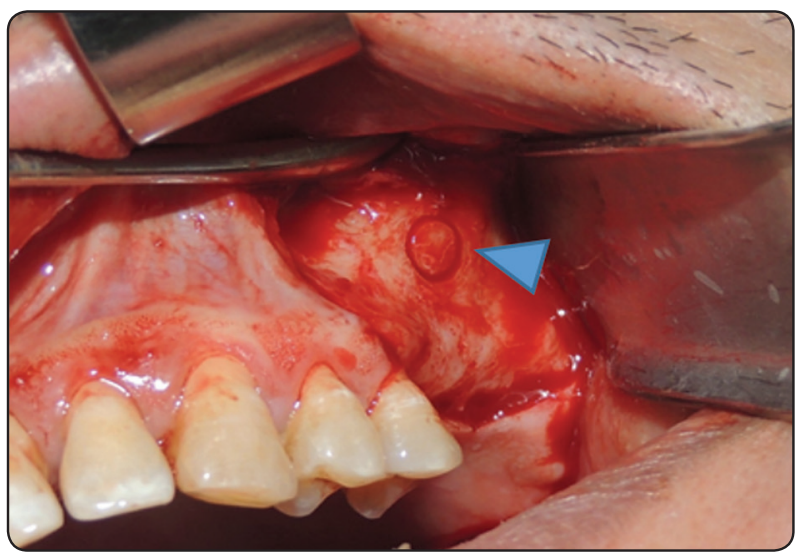

Fig. (2) Image showing flap with buccal trephination (arrowed).

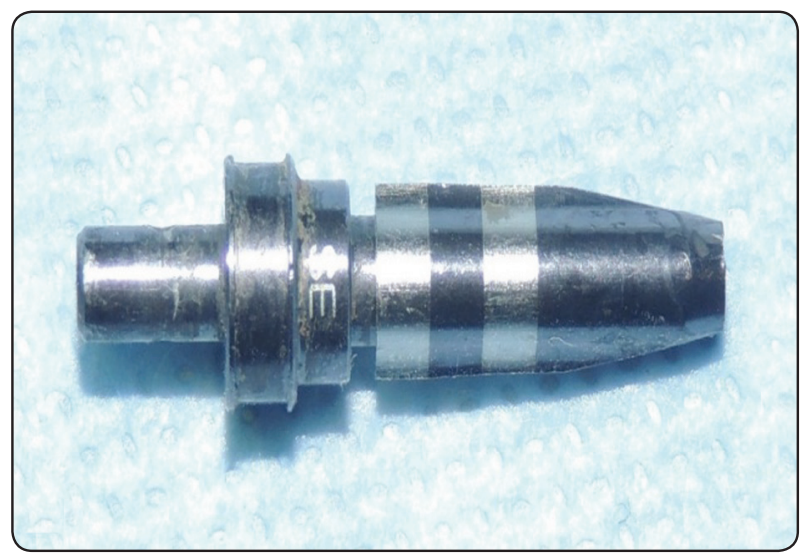

Fig. (4) The Magic Sinus Lifter 
The second surgeon observed all the procedures through direct visualization by using the endoscopic apparatus from the lateral wall of the maxillary sinus to monitor and guid the first surgeon during the sinus membrane elevation in order to achieve good synchronization together.

After finishing the elevation of the schneiderian membrane and the periosteom, the second surgeon removed the endoscope from the lateral wall of the maxillary sinus and re-inserted it from the crestal osteotomy site to be sure there is no any perforation from the lifter that might occurred during the lifting procedure. The implant was inserted by the first surgeon to maintain membrane elevation as no bone graft was added, while the second surgeon endoscopically monitored the process of implant insertion that lifts the membrane through the lateral wall of the maxillary sinus. The small trephined part was returned back to its orginal position to close the window performed in the buccal wall and soft tissue closed with interrupted sutures (Fig. 5-6)

\section{Statistical Analysis}

Quantitative data were presented as mean, standard deviation (SD), median and range values. Membrane thickness data showed non-parametric distribution. Kruskal-Wallis test was used to compare between the three morphological patterns.
Kruskal-Wallis test is a non-parametric statistical method used to determine if there are statistically significant differences between two or more groups of an independent variable.Qualitative data were presented as frequencies and percentages. The significance level was set at $\mathrm{P} \leq 0.05$. Statistical analysis was performed with SPSS ${ }^{\circledR}$ Statistics Version 16 for Windows.

\section{RESULTS}

A total of 19 implants ranging in length from 10 to $13 \mathrm{~mm}$ \& diameter from 3.7 to $5 \mathrm{~mm}$ were placed in 12 patients using crestal approach with a controlled sinus floor elevation without adding any bone grafts depending on the osteogenic property of the Schneiderian memberane and the bony walls of the sinus to form new natural bone.

\section{Clinical results}

All the cases did not show any nasal bleeding or obstruction immediately or even latter within follow ups.

Two cases showed small drops of blood inside the sinus cavity due to minor cutting of the membrane and it is managed immediately by washing the sinus with normal saline solution.

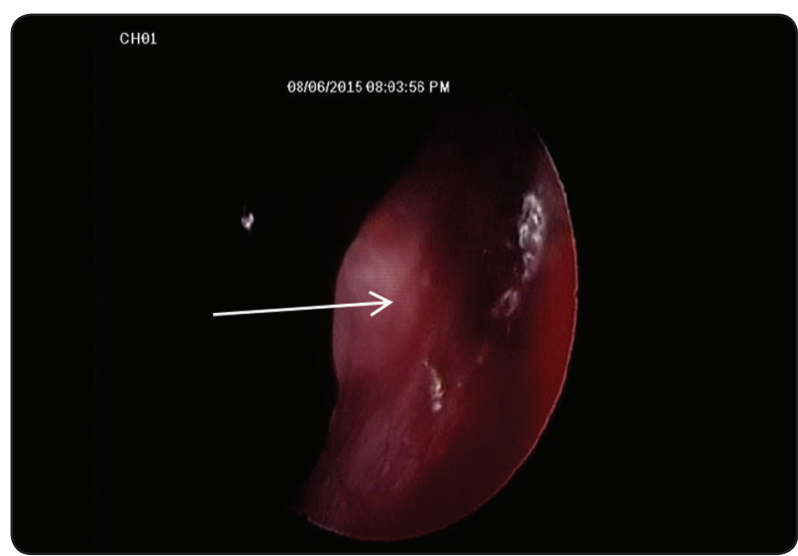

Fig. (6) Membrane elevation shown by endoscope (Arrow). 


\section{Radiographic results}

Pre-operative measurements range was 3-5 mm and the range of elevation of the sinus membrane was 5-8 mm while, the bone formed around implant in range 3-6 $\mathrm{mm}$. Bone deposition around the implant but not over its apex arrowed as showen in (Fig .7).

This appeared as an increase in radio-opacity mesial and distal to all the dental implants inserted after maxillary sinus membrane elevation when compared to immediate postoperative radiographs. Perforation of the schneiderian membrane occurred in 2 cases as shown in (Table 3) and ( Fig. 8). They were closed with platelet rich plasma (PRP) that is inserted from the crestal osteotomy site. The plasma PRP which prepared from patient venous blood sample that was centrifuged to separate PRP which was placed from the crestal osteotomy site to cover perforation before implant placement.

The perforation was noticed in the first case when the endoscope was entered through the osteotomy site of the crest, but it was not observed when the endoscope was inserted through the trephined buccal small widow. The other perforation was observed endoscopically from both approached the crestal and lateral. The perforation was repaired with coverage by PRP and re-evaluated again with the endoscope to reassess the perforation closure.

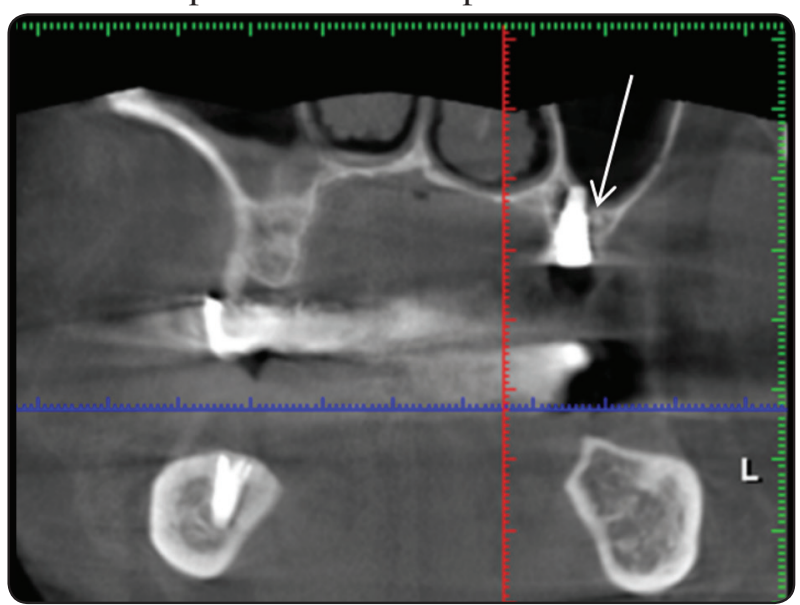

Fig. (7) A CBCT image showing that implant apex is not covered with bone (arrowed).

\section{Membrane thickness and perforation rate}

All cases were classified according to their radiographic membrane thickness and morphology into 3 groups (flat, polyp and irregular) as shown (Fig.9) and compared in Table (1).

There was a statistically significant difference between mean membrane thicknesses with different morphologies (P-value $=0.008)$. Pair-wise comparisons between the three morphological patterns revealed that polyp showed the statistically significantly highest mean membrane thickness. There was no statistically significant difference between flat and irregular membranes; both showed statistically significantly lower mean membrane thickness than polyp as shown in Table 2.

TABLE (1) All cases classification according to membrane morphology shown in the CBCT.

\begin{tabular}{|c|c|c|c|c|}
\hline Case & $\begin{array}{c}\text { Membrane } \\
\text { thickness }\end{array}$ & Flat & Polyp & Irregular \\
\hline 1 & 1.9 & $\mathrm{X}$ & & \\
\hline 2 & 1.6 & $\mathrm{X}$ & & \\
\hline 3 & 0.9 & & & $\mathrm{x}$ \\
\hline 4 & 6.5 & & $\mathrm{x}$ & \\
\hline 5 & 7.8 & & $\mathrm{x}$ & \\
\hline 6 & 7.7 & & $\mathrm{X}$ & $\mathrm{x}$ \\
\hline 7 & 4.2 & $\mathrm{X}$ & & $\mathrm{x}$ \\
\hline 8 & 10.4 & & & \\
\hline 9 & 4.5 & & & \\
\hline 10 & 3.7 & & & \\
\hline 11 & 0.8 & $\mathrm{X}$ & & \\
\hline 12 & 2.20 & & & \\
\hline
\end{tabular}


TABLE (2) Descriptive statistics, results of Kruskal-Wallis and Mann-Whitney U tests for comparison between membrane thickness of different morphologies.

\begin{tabular}{|c|c|c|c|}
\hline Membrane morphology & Mean \pm SD $(\mathrm{mm})$ & Median (Range) & P-value \\
\hline Flat $(\mathrm{n}=4)$ & $2.12 \pm 1.45$ & $1.75(0.8-4.20)$ & \multirow{2}{*}{$0.008^{*}$} \\
\cline { 1 - 3 } Irregular $(\mathrm{n}=4)$ & $2.83 \pm 1.64$ & $7.75(6.50-10.40)$ & \\
\hline
\end{tabular}

*: Significant at $P \leq 0.05$, Different superscripts are statistically significantly different

TABLE (3) Descriptive results of all cases classification according to residual bone hights and the gained bone heights as well the comments for each case.

\begin{tabular}{|c|c|c|c|c|}
\hline Patient no. & $\begin{array}{c}\text { No. of placed } \\
\text { implants }\end{array}$ & $\begin{array}{c}\text { Residual bone } \\
\text { height in } \mathrm{mm}\end{array}$ & $\begin{array}{c}\text { Gained bone } \\
\text { height in } \mathrm{mm}\end{array}$ & Complications \&comments \\
\hline 1 & 2 & 3.7 & 4.1 & Thin membrane \\
\hline 2 & 1 & 4.5 & 3.3 & Whin membrane \\
\hline 3 & 1 & 3.9 & 3.6 & Inadequate primary stability \\
\hline 4 & 1 & 3.8 & 3.4 & Good bone width\& height \\
\hline 5 & 2 & 4.8 & 4.5 & Unseen elevation of the membrane \\
\hline 6 & 1 & 4.2 & 3.2 & Wider implant was required \\
\hline 7 & 2 & 3.7 & 4.8 & Inadequate primary stability \\
\hline 12 & 1 & 4.9 & 4.7 & Thin membrane \\
\hline 10 & 2 & 4.7 & Thin membrane \\
\hline
\end{tabular}




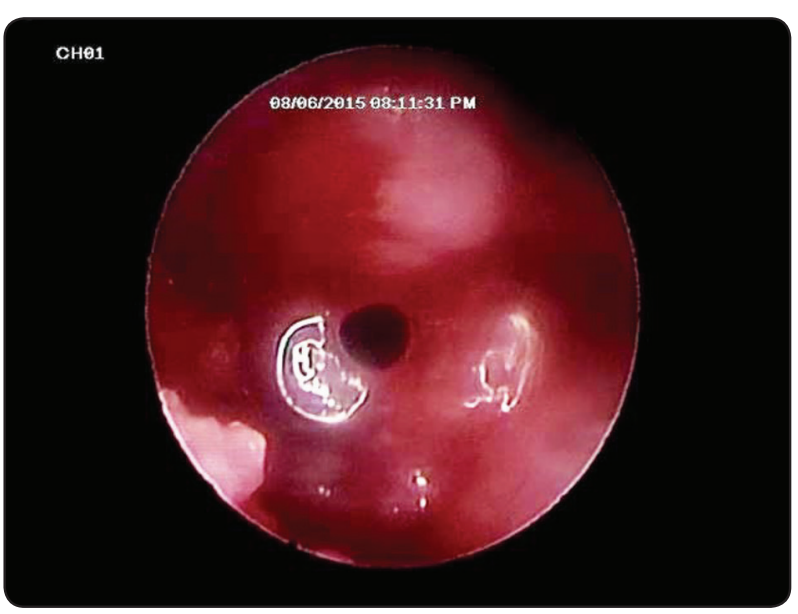

Fig. (8) Minor perforation appeared from crestal osteotomy site by the endoscope.

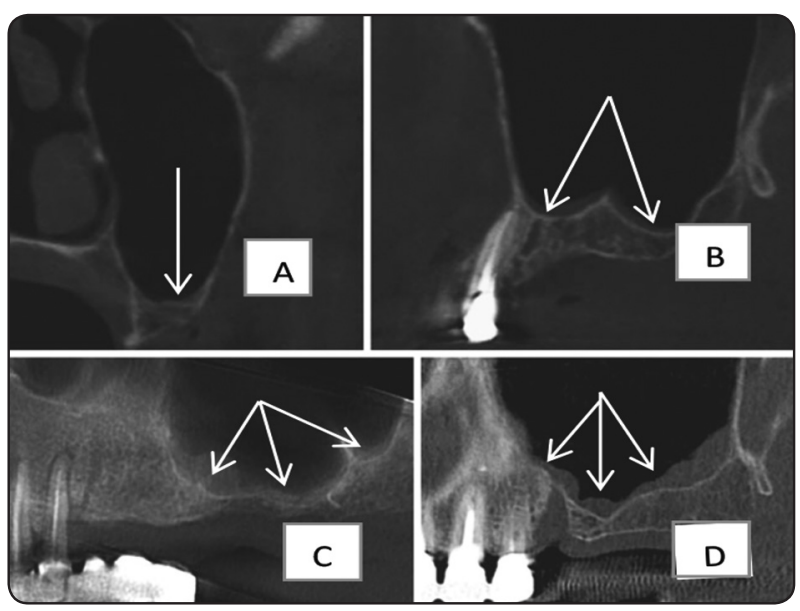

Fig. (9) The schnidern membrane appearancein the CBCT images: A) Normal mucosa. B) Flat mucosal thickening. D) Irregular thickening. And C) Spherical thickening. (All arrowed).

\section{DISCUSSION}

Crestal sinus lifting technique is simple and less invasive procedures, but may result a maxillary sinus membrane perforation especially with limited bone height. ${ }^{1,5}$ In our study we used the endoscope as it a unique dependable method in assessment of Schneiderian membrane perforation precisely.

Up to our knowledge, we made and performed a new immediate visual technique to get access to the maxillary sinus through its lateral wall directly. The trephine bur on hand rotary was used to make a window in the lateral sinus wall for entrance of the endoscope. We found that this procedure has many advantages as less traumatic with minimal heat generation and preserving the trephined buccal bone to reposition it again after endoscopic visualization of sinus membrane. Repositioning this small part of bone has omitted the use of any bone graft or membrane ${ }^{19}$. Our unique technique is very simple, gived marvelous healing \& no bone loss. Moreover, it can advance the trephine bur slowly with a full control even in very thin bone which ultimately assures non-traumatic penetration.

Simultaneous implant placement with sinus floor elevation procedure in the presence of residual bone height of about 3-5 mm could be sufficient to get initial implant primary stability. That is in contrast to Summers ${ }^{2}$ who recommended his technique for patients with at least 5.0 to $6.0 \mathrm{~mm}$ of alveolar bone below the sinus floor. ${ }^{6}$

In our study, we omitted osteotomy site drilling to prevent heat generation, preserve the bone, save time and accelerate osseointegration. We found that the magic splitter is sharp enough to penetrate the alveolar bone below the sinus with less trauma to the bone with very gentle malleting. If surgical drilling was done it would increase bone temperature. The threshold temperature at which heat necrosis of bone occurs is $47^{\circ}$ Celsius/minute. ${ }^{7}$

In the present study, sinus elevation from the crestal approach utilizing the magic sinus lifter ensured a highly tented sinus membrane and space for bone regeneration maintained by the simultaneously placed implant without the use of autogenous bone graft or other alloplastic bone substitute materials. This challenges the utility of conventional approach involving placement of grafting materials into a sinus space created either by the trapdoor window method or by osteotome sinus floor elevation used in the studies of Tong et al ${ }^{8}$, Fabbro et $\mathrm{al}^{9}$, and Garg ${ }^{10}$. Many authors 
have attributed implant failure directly to the sinus membrane perforation ${ }^{11-14}$. Janner et $\mathrm{al}^{17}$ showed that the thickness of the Schneiderian membrane exhibited a wide range, with a minimum value of $0.16 \mathrm{~mm}$ and a maximum value of $34.61 \mathrm{~mm}$.

Sinus elevation utilizing magic sinus lifter technique assisted by endoscope simultaneously with implant placement was found to be a reliable technique as it is minimally invasive and safe. However, Summers ${ }^{2}$ who used the traditional techniques for sinus lifting recorded some complications such as membrane perforation and post-operative infection. Magnification of the field by the endoscope was very useful in visualizing any perforation of the Schneiderian membrane that might occur during its lifting either by the instrumentation or by the implant insertion. Although perforation cannot be avoided, this technique is less invasive than the lateral window technique. It cannot be always recommended as a standard procedure in the posterior maxilla because of the additional equipment needed, cost and the technically demanding procedure.

The standard traditional technique in preparing the osteotomy site for implant placement using the implant drills. In current study, we omitted drills in preparing the osteotomy site completely and used the mallet to allow both the splitter \& magic sinus lifter for penetrating the alveolar bone.

Regarding the technique of trephining the lateral wall of the sinus to get access for the endoscope, Nkenke et $\mathrm{al}^{16}$ in 2002, used a sharp trocar to penetrate the lateral maxillary sinus wall to be able to introduce the endoscope inside the sinus. After we had used Nkenke et $\mathrm{al}^{16}$ technique, we found that his technique accomplish the surgical field with unclear bloody field due to the trauma that occurred from the sharp trocar he had used. In our study, we used a trephine bur on hand rotary drill to get a gentle cutting with a full control, beside preserving the bone that was cut and preposition it again into its original place back after finishing the procedure as a noval technique.

\section{CONCLUSION}

The advantages offered by the newly designed osteotome made the technique simple, fast plus omitting the use of drills. With the endoscopically controlled osteotome sinus floor elevations, perforations of the sinus membrane can be visualized; however, they cannot be avoided. Although this technique is less invasive than the lateral window technique, it cannot be recommended as a standard procedure in the posterior maxilla because of the additional equipment needed and the technically demanding procedure. The low invasive technique requires a higher level of surgical training, senqronized hands and experience in endoscopic imaging.

\section{ACKNOWLEDGEMENT}

We acknowledge the role of Prof. K. Barakat for providing his own endoscope in performing most of the practical endoscopy. As well Dr. Samy Elian for his collaboration.

\section{Conflict of interest:}

The Authors declare No conflict of interests.

\section{REFERENCES}

1. Boyne P, James RA: grafting of maxillary sinus floor with autogenous marrow and bone. J Oral maxillofac Surg 38: 113-119, 1980.

2. Summers RB. A new concept in maxillary implant surgery: The osteotome technigue.Comped Contin Educ Dent; 15: 152-160, 1994.

3. Ardekian L, Oved-Peleg E, Mactei EE, Peled M. The clinical significance of sinus membrane perforation during augmentation of the maxillary sinus. J Oral Maxillofac Surg.64: 277-282, 2006.

4. Pommer B, Frantal S, Willer J, Posch M, Watzek G, Tepper G. Impact of dental implant length on early failure rates: a meta-analysis of observational studies. J Clin Periodontol 38: 856-863, 2011.

5. Zitzmann NU, Scharer P: sinus elevation procedures in the resorbed posterior maxilla. Oral Surg Oral Pathol Oral Radiol Endod 85: 8:14, 1998. 
6. Summers RB. The osteotome technique: part 3: the less invasive methods of elevating the sinus floor. Compend Contin Educ Dent. 15:698-704 , 1994.

7. Esposito, M., Hirsch, J. M., Lekholm, U. \& Thomsen, P. Biological factors contributing to failures of osseointegrated oral implants. (II). Etiopathogenesis. European Journal of Oral Sciences 106, 721-764, 1998.

8. Tong DC, RIOUX K ,Drangsholt M : A review of survival rates for implants placed in grafted maxillary sinuses using meta analysis. Int J Oral Maxillofac Implants. 13:175, 1998.

9. Fabbro MD, Testori T, Francitti L : Systemic review of survival rates for implants placed in the grafted maxillary sinus. Int J periodontics Restorative Dent 24:565, 2004.

10. Garg AK: Augmentation grafting of maxillary sinus for placement of dental implants: Anatomy, physiology, and procedures. Implant Dent 8:36, 1999.

11. Komarnyckyj OG, London RM. Osteotome single stage dental implant placement with and without sinus elevation: a clinical report. Int J Oral Maxilofac Implants. 13:799-804, 1998.

12. Proussaefs P, Olivier HS, Lozada J. Histologic evaluation of a 12-year-old threaded hydroxyapatitecoated implant placed in conjunction with subantral augmentation procedure: a clinical report. J Prosthetic Dent. 92:17-22, 2004.

13. Buchmann R, Khoury F, Faust C, Lange DE. Periimplant conditions in periodontally compromised patients following maxillary sinus augmentation: a long term post therapy trial. Clin Oral Implants Res10: 103-110, 1999.

14. Schwartz-Arad D, Herzberg R, Dolev E. The prevalence of surgical complications of the sinus graft procedure and their impact on implant survival. J Periodontol. 75:511$516,2004$.

15. Fugazzotto PA. Immediate implant placement following a modified trephine/osteotome approach: Success rates of 116 implants through four years in function. Int J Oral Maxillofac Implants. 17(1):113-120, 2002.

16. Nkenke E, Schlegel A, Schultze-Mosgau S, et al. The endoscopically controlled osteotome sinus floor elevation: a preliminary prospective study. Int $\mathrm{J}$ Oral Maxillofac Implants17(4): 557-566, 2002.

17. Janner et al, Characteristics and dimensions of the Schneiderian membrane: a radiographic analysis using cone beam computed tomography in patients referred for dental implant surgery in the posterior maxilla. Clin. Oral Impl. Impl. Res. Xx, :215:219, 2011. , 2011.

18. Soikkonen K, Ainamo A. Radiographic maxillary sinus findings in the elderly. Oral Surg Oral Med Oral Pathol Oral Radiol Endod. 80(4):487-91, 1995.

19. Srouji S, Kizhner T, Ben David D, et al. The Schneiderian membrane contains osteoprogenitor cells: in vivo and in vitro study. Calcif Tissue Int.84:138-145, 2009 